\title{
Suppression of Marangoni convection of silicon melt by a non-contaminating method
}

\author{
Jie $\mathrm{Li}^{\text {a }}$, Mingwei $\mathrm{Li}^{\mathrm{a}, *}$, Wenrui $\mathrm{Hu}^{\mathrm{b}}$, Danling Zeng ${ }^{\mathrm{a}}$ \\ a Institute of Power Engineering, Chongqing University, Chongqing 400044, China \\ ${ }^{\mathrm{b}}$ Institute of Mechanics, CAS, Beijing 100083, China
}

Received 23 January 2003; received in revised form 5 June 2003

\begin{abstract}
A set of numerical simulation of the effect of the gas shearing flow over a silicon melt free surface on Marangoni convection under microgravity condition was conducted by using finite element method. For given gas channel width, Marangoni number and aspect ratio a remarkable reduction of Marangoni convection in silicon liquid bridge can be achieved by choosing the optimal gas velocity in accordance with the correlation proposed in the paper. The effectiveness of the reduction of the gas flow under different conditions shows that, in some cases, Marangoni convection reduction of $99 \%$ can be realized by this non-contaminating method.
\end{abstract}

(c) 2003 Elsevier Ltd. All rights reserved.

Keywords: Marangoni convection; Silicon; Non-contamination method; Finite-element method

\section{Introduction}

It is well known that Marangoni convection (MC) dominating under microgravity gives strong influence on the crystal growth process. Space experiments conducted by Eyer et al. [1] have shown that time-dependent MC will lead to solute segregation which is the underlying cause of striation. The floating-zone method by the combination with microgravity environment is expected a promising containerless method for the growth of striation-free crystal, but the MC must be suppressed effectively. In order to produce high-quality semiconductor, some approaches such as liquid encapsulant method, have been applied to reduce the Marangoni convection [2-10]. Besides, a non-contaminating method [11] to reduce MC was suggested and investigated experimentally by Dressler et al., where a vertical jet of gas (argon) was blown tangentially over the free surface, producing a viscous shearing drag opposing the thermocapillary shear at the surface. A small drop of silicone oil with $1.5 \mathrm{~mm}$

\footnotetext{
${ }^{*}$ Corresponding author. Tel.: +86-23-654-15008; fax: +8623-651-02473/02471.

E-mail address: mwlizao@yahoo.com (M. Li).
}

height was chosen, which was confined in a transparent box $(1.5 \mathrm{~mm} \times 1.5 \mathrm{~mm} \times 2.5 \mathrm{~mm})$ with one vertical side open for the free surface. Their experimental results show that the MC velocity reduction of $66 \%$ can be achieved. In the global simulation of a silicon Czochralski furnace, it was found that the gas flow has a significant influence on the melt convective pattern [12]. However, up to now, there is no investigation report associated with applying this method to suppress the $\mathrm{MC}$ in silicon liquid bridge to our knowledge.

In the present work, a set of numerical simulation to investigate the effect of argon gas viscous shearing flow on $\mathrm{MC}$ in silicon liquid bridge is performed under different gas channel width, Marangoni number and aspect ratio of the height to radius by finite element method. The results show that a significant reduction of Marangoni convection in the silicon melt can be realized.

\section{Model formulation}

\subsection{Physical model}

Consider an axisymmetric silicon melt column contained between two planar disks with a height $H$ apart, 


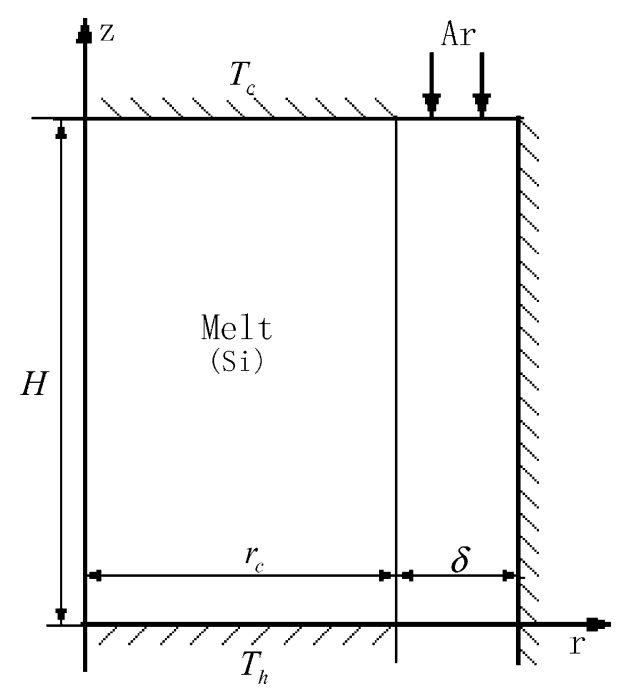

Fig. 1. Physical model.

as shown in Fig. 1. Argon gas flows surrounding the free surface from the cold end to the hot end. Let $r_{\mathrm{c}}$ be the radius of the melt, $\delta$ be the width of the channel. The silicon melt is assumed to be incompressible Newtonian fluid; and the liquid-gas interface is smooth and nondeformable. The endwalls at $z=0, H$ are maintained at constant temperature $T_{\mathrm{h}}$ and $T_{\mathrm{c}}$, respectively. The law of surface tension versus temperature is assumed to be linear:

$\sigma=\sigma_{\mathrm{c}}-\gamma\left(T-T_{\mathrm{c}}\right)$,

in which $\sigma_{\mathrm{c}}$ is the surface tension of the melt-gas interface at temperature $T_{\mathrm{c}}\left(T_{\mathrm{c}}=T_{\mathrm{m}}=1683 \mathrm{~K}\right), \gamma$ is the variation rate of surface tension with temperature $T$, $\gamma=-\partial \sigma / \partial T$. Gravity is assumed to be absent.

\subsection{Mathematical model}

\subsubsection{Governing equations}

In the governing equation, the dimensionless parameters (with asterisks) are adopted which are defined as follows:

$r=r_{\mathrm{c}} r^{*}, \quad z=z^{*} H, \quad \delta=r_{\mathrm{c}} \delta^{*} \quad u_{i}=u_{i}^{*} \frac{v_{1}}{r_{\mathrm{c}}}$

$v_{i}=v_{i}^{*} \frac{v_{1}}{r_{\mathrm{c}}}, \quad p_{i}=\frac{\rho_{1} v_{1}^{2}}{r_{\mathrm{c}}^{2}} p_{i}^{*}, \quad T_{i}=\left(T_{\mathrm{h}}-T_{\mathrm{c}}\right) T_{i}^{*}+T_{\mathrm{c}}$,

$\rho_{i}=\rho_{i}^{*} \rho_{1}, \quad \lambda_{i}=\lambda_{1} \lambda_{i}^{*}, \quad \mu_{i}=\mu_{i}^{*} \mu_{1}, \quad \kappa_{i}=\kappa_{i}^{*} \kappa_{1}$,

where $r$ and $z$ are the radial and the axial coordinate, respectively, $u_{i}$ is the radial velocity components and $v_{i}$ the axial velocity components, $p_{i}$ the pressure, $T_{i}$ the temperature, $\rho_{i}$ the density, $\lambda_{i}$ the thermal conductivity, $v_{1}$ the kinematic viscosity of melt, $\mu_{i}$ the dynamic viscosity and $\kappa_{i}$ the thermal diffusivity, respectively.
Subscripts $i$ indicate Silicon melt $(i=1)$, Argon gas $(i=\mathrm{g})$, respectively.

The dimensionless general governing equations of momentum and energy are given as follow with the asterisk dropped conventionally:

$$
\begin{aligned}
& \frac{1}{r} \cdot \frac{\partial\left(r u_{i}\right)}{\partial r}+\frac{1}{A} \frac{\partial v_{i}}{\partial z}=0 \\
& \rho_{i}\left(u_{i} \frac{\partial u_{i}}{\partial r}+\frac{1}{A} v_{i} \frac{\partial u_{i}}{\partial z}\right)=-\frac{\partial p_{i}}{\partial r}+\mu_{i}\left(\frac{\partial^{2} u_{i}}{\partial r^{2}}+\frac{1}{A^{2}} \frac{\partial^{2} u_{i}}{\partial z^{2}}\right. \\
& \left.+\frac{1}{r} \frac{\partial u_{i}}{\partial r}-\frac{u_{i}}{r^{2}}\right) \\
& \rho_{i}\left(u_{i} \frac{\partial v_{i}}{\partial r}+\frac{1}{A} v_{i} \frac{\partial v_{i}}{\partial z}\right) \\
& =-\frac{1}{A} \frac{\partial p_{i}}{\partial z}+\mu_{i}\left(\frac{\partial^{2} v_{i}}{\partial r^{2}}+\frac{1}{A^{2}} \frac{\partial^{2} v_{i}}{\partial z^{2}}+\frac{1}{r} \frac{\partial v_{i}}{\partial r}\right), \\
& \operatorname{Pr}_{1}\left(u_{i} \frac{\partial T_{i}}{\partial r}+\frac{1}{A} v_{i} \frac{\partial T_{i}}{\partial z}\right)=\kappa_{i}\left(\frac{\partial^{2} T_{i}}{\partial r^{2}}+\frac{1}{r} \frac{\partial T_{i}}{\partial r}+\frac{1}{A^{2}} \frac{\partial^{2} T_{i}}{\partial z^{2}}\right),
\end{aligned}
$$

where Prandtl number is defined by $\operatorname{Pr} r_{1}=\rho_{1} c_{\mathrm{pl}} v_{1} / \lambda_{1}, c_{\mathrm{pl}}$ the specific heat of melt, and $A$ the aspect ratio $A=H / r_{\mathrm{c}}$.

\subsubsection{Boundary conditions}

At the cold endwall of the melt:

$u_{1}=v_{1}=0, \quad T_{1}=0$.

At the inlet of the gas channel:

$u_{\mathrm{g}}=0, \quad v_{\mathrm{g}}=v_{\text {in }}, \quad T_{\mathrm{g}}=0$.

At the hot endwall of the melt:

$u_{1}=v_{1}=0, \quad T_{1}=1$.

At the side wall of the gas channel:

$u_{\mathrm{g}}=v_{\mathrm{g}}=0, \quad T_{\mathrm{g}}=T(z)$.

At the melt/gas interface:

$u_{1}=0, \quad u_{\mathrm{g}}=0, \quad v_{\mathrm{l}}=v_{\mathrm{g}}$,

$T_{1}=T_{\mathrm{g}}$,

$-\lambda_{1} \frac{\partial T_{1}}{\partial r}=-\lambda_{\mathrm{g}} \frac{\partial T_{\mathrm{g}}}{\partial r}$,

$\frac{\partial u_{1}}{\partial r}-\mu_{\mathrm{g}} \frac{\partial u_{\mathrm{g}}}{\partial r}=-M a \cdot \frac{1}{A} \frac{\partial T}{\partial z}$,

where $v_{\text {in }}$ is the dimensionless inlet gas velocity (hereafter this is called gas velocity for short). The dimensionless Marangoni number $\mathrm{Ma}$ in equation is defined as follows:

$M a=\frac{\Delta T \cdot r_{\mathrm{c}} \gamma}{\mu_{1} v_{1}}$,

here $\Delta T=T_{\mathrm{h}}-T_{\mathrm{c}}$. 
The numerical procedure used here and the related physical properties needed in the calculation can be found in Ref. [12].

\section{Results}

A set of numerical simulation of flow and heat transfer in both melt and gas phases for different gas velocity in the case of $\delta=0.3, A=2.0$ and $M a=$ 108,000 was conducted. The suppressive effect of gas flow on the melt convection was observed from the results obtained. Because of the length limit of the paper, only two figures of the contours of stream function and isothermals of the silicon melt and the gas with two different gas velocity are presented in Figs. 2 and 3, respectively. It should be emphasized that the low gas velocity gives a little influence on MC, a large counter clockwise Marangoni convection vortices still occupy the bulk melt. The highest velocity of the melt occurs at

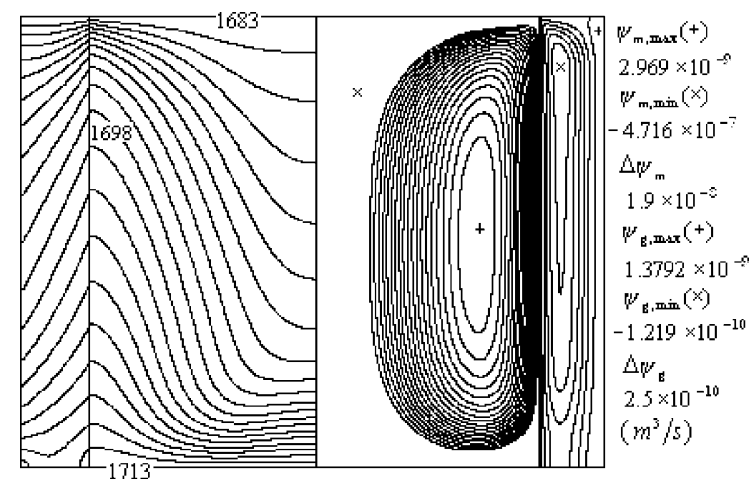

Fig. 2. Isotherms (left, $\Delta T=1.5 \mathrm{~K}$ ) and contours of stream function of melt and gas (right) with $A=2.0, \delta=0.3$, $M a=1.08 \times 10^{5}, v_{\text {in }}=500$.

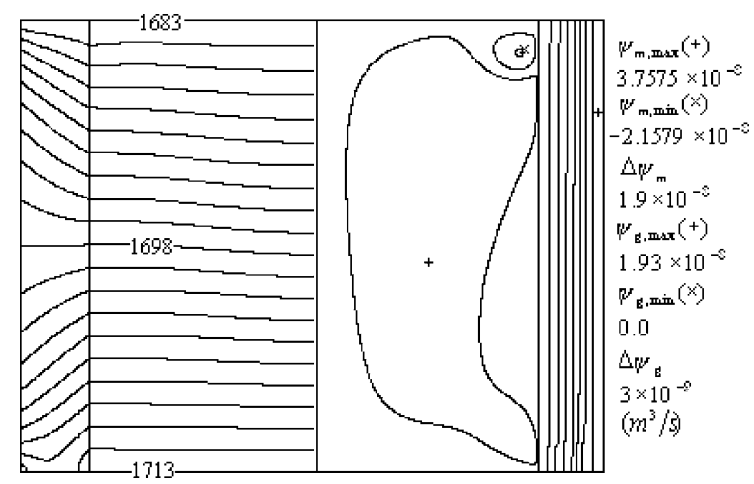

Fig. 3. Isotherms (left, $\Delta T=1.5 \mathrm{~K}$ ) and contours of stream function of melt and gas (right) with $A=2.0, \delta=0.3$, $M a=1.08 \times 10^{5}, v_{\text {in }}=24,300$. the melt/gas interface. The distorted isothermal depicted that convection heat transfer is dominated in the melt. In this case, due to the continuity conditions must be satisfied at the melt/gas interface, the strong Marangoni convection results in an upward flow of gas phase in the adjacent of the melt/gas interface. With gradually increasing gas velocity, the induced upward gas flow slows down and eventually dies away. Inversely, viscous shearing drag of gas acting on the melt surface makes Marangoni convection become weaker and weaker, as the gas velocity goes to a certain value, in the present case, when $v_{\text {in }}=24,300$, i.e. $67.23 \mathrm{~cm} / \mathrm{s}$, the dimensionless average velocity of the melt $v_{\mathrm{av}, \mathrm{g}}$ reaches the minimum value, i.e. $v_{\mathrm{av}, \mathrm{g}}=30.64,0.085 \mathrm{~cm} / \mathrm{s}$. Here $v_{\mathrm{av}, \mathrm{g}}$ stands for the non-dimensional average velocity of the melt defined by $v_{\mathrm{av}, \mathrm{g}}=\sum\left(\sqrt{u_{i}^{2}+v_{i}^{2}}\right) / N$, in which $N$ represents the total number of the nodes in the region of the melt. After the minimum value has reached, the dimensionless average velocity increases again and the melt flow pattern tends to be more complicated. Therefore, we can conclude that there exists an extreme value of the non-dimensional average velocity of the melt which reflects the weakest flow of the melt. Undoubtedly, an effective suppression can be achieved by properly choosing the gas velocity for a specified condition.

To quantitatively evaluate the effectiveness of flow reduction caused by gas flow on the melt convection for different gas channel width $\delta$, Marangoni number $M a$ and aspect ratio $A$, a parameter PR called reduction percentage was introduced:

$\mathrm{PR}=\frac{-\left(v_{\mathrm{av}, \mathrm{g}}-v_{\mathrm{av}}\right)}{v_{\mathrm{av}}} \times 100 \%$,

where $v_{\mathrm{av}}$ denotes the non-dimensional average velocity of the melt without gas flow. Fig. 4 indicates the change of the non-dimensional average velocity of the melt $v_{\mathrm{av}, \mathrm{g}}$

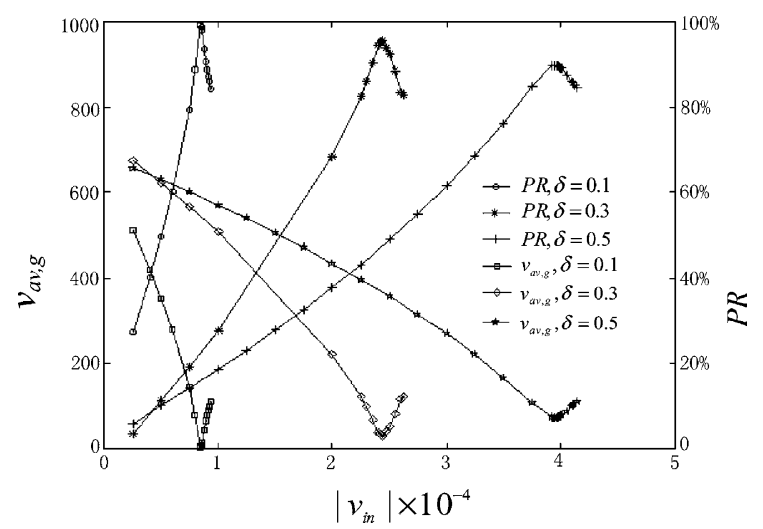

Fig. 4. Average velocity $v_{\mathrm{av}, \mathrm{g}}$ and reduction percentage PR versus gas velocity $v_{\text {in }}$ for various gas channel width $\delta$ with $A=2.0, M a=1.08 \times 10^{5}$. 


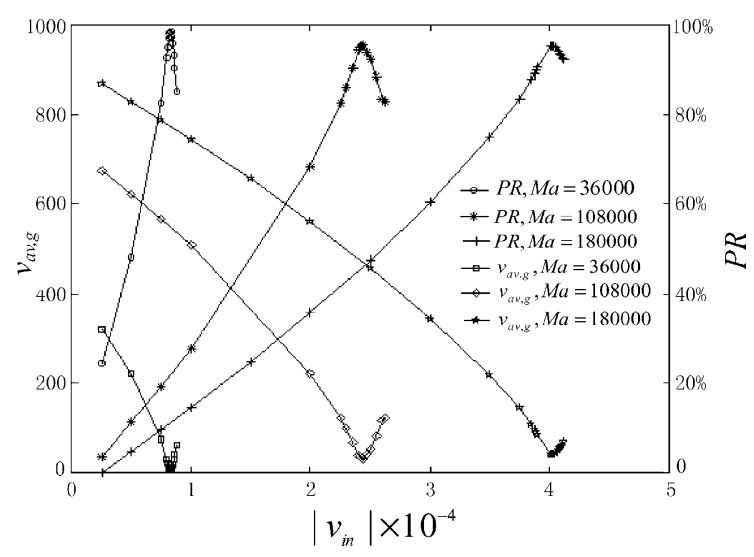

Fig. 5. Average velocity $v_{\mathrm{av}, \mathrm{g}}$ and reduction percentage $\mathrm{PR}$ versus gas velocity $v_{\text {in }}$ for various Marangoni number $M a$ with $A=2.0, \delta=0.3$.

and reduction percentage PR with the gas velocity $v_{\text {in }}$ for various gas channel width $\delta$. The values of $v_{\text {in }}$ and PR corresponding to extreme points of the curves $\mathrm{PR}-v_{\text {in }}$ are nothing but the optimal gas velocity $\left(v_{\text {in,opt }}\right)$ and reductive effectiveness $\left(\mathrm{PR}_{b}\right)$, respectively. The value of $v_{\text {in,opt }}$ decreases from $39,500(109.3 \mathrm{~cm} / \mathrm{s})$ to $8450(23.34 \mathrm{~cm} / \mathrm{s})$ and the value of $\mathrm{PR}_{b}$ increases from $89.82 \%$ to $99.47 \%$ when reducing the value of $\delta$ from 0.5 to 0.1 , this means that narrowing the width of the gas channel is beneficial for suppressing Marangoni convection. This is consistent qualitatively with the result of Ref. [8].

In the situation of the fixed gas channel width and aspect ratio, a higher optimal gas velocity $v_{\text {in,opt }}$ is required, meanwhile the value of $\mathrm{PR}_{b}$ has a slightly decrease with increasing Marangoni number $\mathrm{Ma}$ (see Fig. 5).

For different aspect ratio $A$, the values of $v_{\text {in,opt }}$ and $\mathrm{PR}_{b}$ are different, as shown in Fig. 6. The change of the

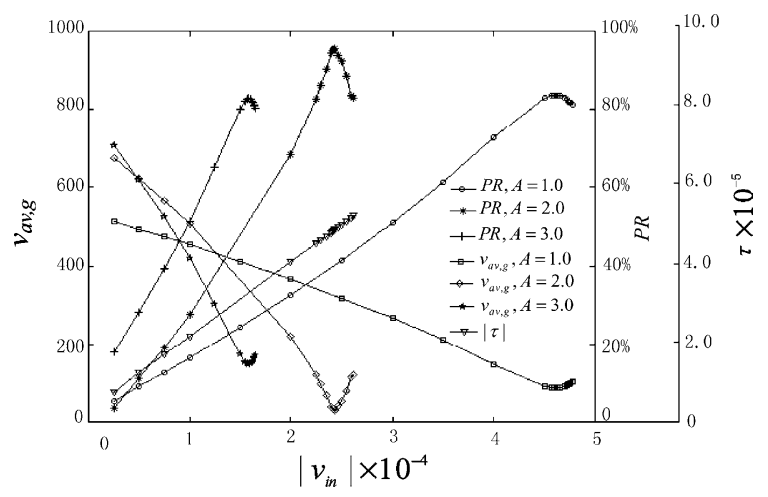

Fig. 6. Average velocity $v_{\mathrm{av}, \mathrm{g}}$, reduction percentage PR and shearing stress $\tau$ versus gas velocity $v_{\text {in }}$ for various aspect ratio $A$ with $\delta=0.3, M a=1.08 \times 10^{5}$.
$\mathrm{PR}_{b}$ and $v_{\text {in,opt }}$ with $A$ is not monotonous. When $A=2.0$, the value of $\mathrm{PR}_{b}$ becomes the highest, $\mathrm{PR}_{b}=95.62 \%$. Meanwhile one can find that the shearing stress acting on the silicon surface increases linearly as the gas velocity increases for the fixed values of $\delta, A$ and $M a$.

From the numerical result obtained, the optimal gas velocity can be expressed in the following correlation:

$v_{\mathrm{opt}}=1.762 \cdot \delta^{0.9562} \cdot A^{-0.9655} \cdot M a^{0.9788}$

with the correlative coefficient of 0.99 . This correlation provides us an approximate choice of the gas velocity so that the highest degree of reduction of Marangoni convection can be realized.

\section{Conclusions}

A non-contaminating method for suppressing Marangoni convection in a silicon liquid bridge was realized by introducing a tangential flow of Argon gas passing over the silicon melt free surface, thus to form a viscous shear drag opposing to Marangoni shearing at the free surface. Numerical simulation using finite element method was conducted to demonstrate the effectiveness of the approach. The effectiveness of the reduction of the flow is associated with the inlet gas velocity for given conditions. By properly choosing the gas velocity, the optimal reductive effectiveness of Marangoni convection can be achieved. The optimal reductive effectiveness under different gas channel width, Marangoni number and aspect ratio has been presented. It is proved that the MC reduction of $99 \%$ can be realized in some situation.

\section{Acknowledgements}

This work was supported by the Excellent Young Teachers Program of MOE (EYTP), P.R.C. [2002] 40 and by National Microgravity Laboratory, Institute of Mechanics, Chinese Academy of Science.

\section{References}

[1] A. Eyer, H. Leiste, R. Nitsche, Float zone growth of silicon under microgravity in a sounding rocket, J. Cryst. Growth 71 (1985) 173-182.

[2] A. Eyer, H. Leiste, Striation-free silicon crystal by floatzoning with surface-coated melt, J. Cryst. Growth 71 (1985) 249-252.

[3] T. Doi, J. Koster, Marangoni convection in two immiscible liquid layers subject to a horizontal temperature gradient, in: Proceedings of the 7th International Conference on Physico-chemical Hydrodynamics, MIT, Cambridge, MA, 1989. 
[4] E. Crespo del Arco, G.P. Extrement, R.I. Sani, Thermocapillary convection in a two-layer fluid system with flat interface, Adv. Space Res. 11 (1991) 129-132.

[5] J. Li, J. Sun, Z. Saghir, Buoyant and thermocapillary flow in liquid encapsulated float zone, J. Cryst. Growth 131 (1993) 83-96.

[6] Q.S. Liu, G. Chen, B. Roux, Thermogravitational and thermocapillary convection in a cavity containing two superposed immiscible liquid layers, Int. J. Heat Mass Transfer 36 (1993) 101-117.

[7] J. Li, M.Z. Saghir, Thermocapillary convection in liquid encapsulation float zone, in: Int. Symp. On Microgravity Science and Application, Beijing, China, 1993.

[8] M. Li, D. Zeng, The effect of liquid encapsulation on the Marangoni convection in a liquid column under micro- gravity condition, Int. J. Heat Mass Transfer 39 (17) (1996) 3725-3732.

[9] Q.S. Liu, B. Roux, M.G. Velarde, Thermocapillary convection in two-layer systems, Int. J. Heat Mass Transfer 41 (11) (1998) 1499-1511.

[10] M. Li, D. Zeng, T. Zhu, Instability of the Marangoni convection in a liquid bridge with liquid encapsulation under microgravity condition, Int. J. Heat Mass Transfer 45 (2002) 157-164.

[11] R.F. Dressler, N.S. Sivakumaran, Non-contaminating method to reduce Marangoni convection in microgravity float-zones, J. Cryst. Growth 88 (1988) 148-158.

[12] M. Li, Y. Li, N. Imaishi, T. Tsukada, Global simulation of a silicon Czochralski furnace, J. Cryst. Growth 234 (2002) $32-46$. 